\title{
Three-wave coupling coefficients for perpendicular wave propagation in a magnetized plasma
}

\author{
G. Brodin and Lennart Stenflo \\ Linköping University Post Print
}

\section{Tweet}

N.B.: When citing this work, cite the original article.

Original Publication:

G. Brodin and Lennart Stenflo, Three-wave coupling coefficients for perpendicular wave propagation in a magnetized plasma, 2015, Physics of Plasmas, (22), 10.

http://dx.doi.org/10.1063/1.4934938

Copyright: American Institute of Physics (AIP)

http://www.aip.org/

Postprint available at: Linköping University Electronic Press

http://urn.kb.se/resolve?urn=urn:nbn:se:liu:diva-123160 


\section{AIP | Physics of

\section{Three-wave coupling coefficients for perpendicular wave propagation in a magnetized plasma}

G. Brodin and L. Stenflo

Citation: Physics of Plasmas 22, 104503 (2015); doi: 10.1063/1.4934938

View online: http://dx.doi.org/10.1063/1.4934938

View Table of Contents: http://scitation.aip.org/content/aip/journal/pop/22/10?ver=pdfcov

Published by the AIP Publishing

\section{Articles you may be interested in}

Kinetic theory of weak turbulence in magnetized plasmas: Perpendicular propagation

Phys. Plasmas 22, 082310 (2015); 10.1063/1.4928380

Dust-acoustic shock waves in a charge varying electronegative magnetized dusty plasma with nonthermal ions: Application to Halley Comet plasma

Phys. Plasmas 20, 103704 (2013); 10.1063/1.4825240

Dispersion relations of nonlinearly coupled electromagnetic and electrostatic waves in relativistic plasmas

Phys. Plasmas 17, 012305 (2010); 10.1063/1.3292648

Obliquely propagating low frequency electromagnetic shock waves in two dimensional quantum magnetoplasmas

Phys. Plasmas 16, 042314 (2009); 10.1063/1.3119209

Nonlinear interaction of dispersive Alfvén waves and magnetosonic waves in space plasma

Phys. Plasmas 16, 032901 (2009); 10.1063/1.3074788

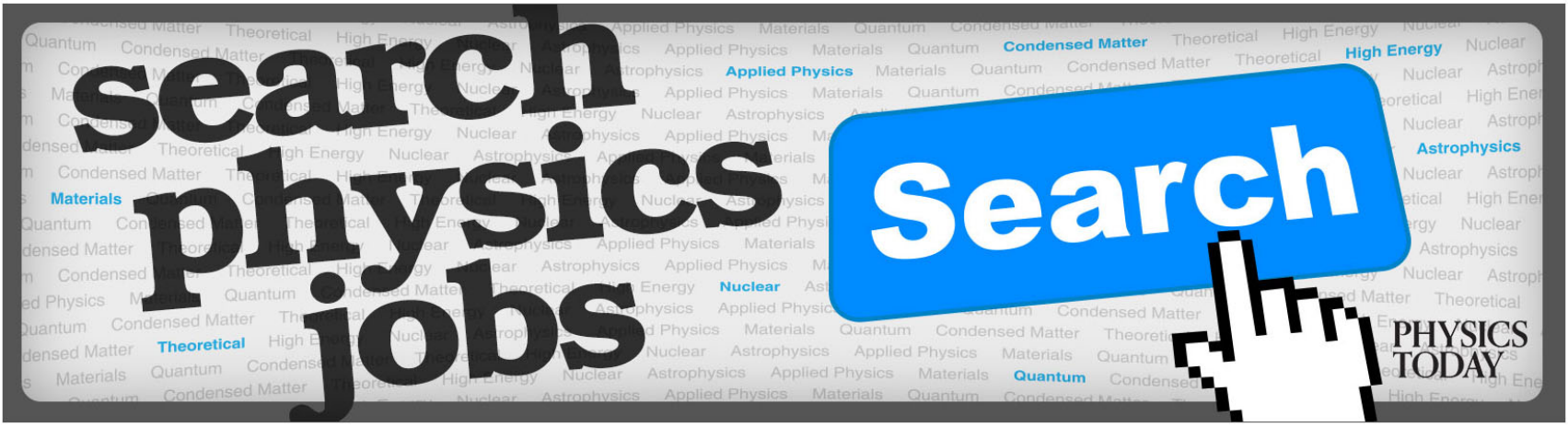




\title{
Three-wave coupling coefficients for perpendicular wave propagation in a magnetized plasma
}

\author{
G. Brodin ${ }^{1}$ and L. Stenflo ${ }^{2}$ \\ ${ }^{1}$ Department of Physics, Umea University, SE-901 87 Umeå, Sweden \\ ${ }^{2}$ Department of Physics, Linköping University, SE-581 83 Linköping, Sweden
}

(Received 11 September 2015; accepted 19 October 2015; published online 29 October 2015)

The resonant interaction between three waves in a uniform magnetized plasma is reconsidered. Starting from previous kinetic expressions, we limit our investigation to waves propagating perpendicularly to the external magnetic field. It is shown that reliable results can only be obtained in the two-dimensional case, i.e., when the wave vectors have both $x$ and $y$ components. (C) 2015 AIP Publishing LLC. [http://dx.doi.org/10.1063/1.4934938]

The theory for wave-wave interactions in plasmas has up to now been developed during more than 50 years. Almost all kinds of possible three-wave coupling phenomena have thus been described in numerous papers. This theory has many applications, for example, in laser-fusion research (e.g., Ref. 1) and later also in ionospheric plasma studies (e.g., Refs. 2-6). A previous review paper, ${ }^{7}$ with many references to those early papers, covers the first 30 years of that activity. Nonlinear plasma physics has however also flourished during the recent two decades, and several complementary studies have thus been published, including quantum properties, such as particle dispersive effects (e.g., Refs. 8 and 9) and/or degeneracy effects ${ }^{10}$ as well as bounded plasmas, e.g., Ref. 11. In addition, Yoon recently ${ }^{12}$ investigated in detail the limit of one-dimensional wave propagation perpendicular to the external magnetic field. The purpose of the present Brief Communication is to demonstrate that it is easy to generalize the analysis to consider two-dimensional wave propagation. This is a necessary prerequisite to reliable comparisons with future experiments.
The general case of three-wave interactions in a uniform plasma situated in an external constant magnetic field $B_{0} \hat{\mathbf{z}}$ has been considered in many previous papers. The resonance conditions for the frequencies $\omega_{j}(j=1,2,3)$ and wavevectors $\mathbf{k}_{j}(j=1,2,3)$ have then been supposed to be satisfied, i.e., $\omega_{3}=\omega_{1}+\omega_{2}$ and $\mathbf{k}_{3}=\mathbf{k}_{1}+\mathbf{k}_{2}$. When calculating the coupling coefficients, it turns out that they contain a common factor V. It is therefore possible to write the three coupled equations as (e.g., Refs. 7, 13, 14)

$$
\frac{d W_{1,2}}{d t}=-2 \omega_{1,2} \operatorname{ImV}
$$

and

$$
\frac{d W_{3}}{d t}=2 \omega_{3} \operatorname{ImV}
$$

where $W=\varepsilon_{0} \mathbf{E}^{*} \cdot(1 / \omega) \partial\left(\omega^{2} \boldsymbol{\varepsilon}\right) \mathbf{E}$ is the wave energy, $\mathbf{E}$ is the electric field amplitude, $\boldsymbol{\varepsilon}$ is the usual textbook dielectric tensor, ${ }^{15}$ and ImV stands for the imaginary part of $\mathrm{V}$, where ${ }^{7}$

$$
\begin{aligned}
\mathbf{V}= & \sum_{s} m \int d \mathbf{v} F_{0}(\mathbf{v}) \sum_{\substack{p_{1}+p_{2}=p_{3} \\
p_{j}=0, \pm 1, \pm 2, \ldots}} I_{1}^{p_{1}} I_{2}^{p_{2}} I_{3}^{-p_{3}} \\
& \times\left[\frac{\mathbf{k}_{1} \cdot \mathbf{u}_{1 p_{1}}}{\omega_{1 d}} \mathbf{u}_{2 p_{2}} \cdot \mathbf{u}_{3 p_{3}}^{*}+\frac{\mathbf{k}_{2} \cdot \mathbf{u}_{2 p_{2}}}{\omega_{2 d}} \mathbf{u}_{1 p_{1}} \cdot \mathbf{u}_{3 p_{3}}^{*}+\frac{\mathbf{k}_{3} \cdot \mathbf{u}_{3 p_{3}}^{*}}{\omega_{3 d}} \mathbf{u}_{1 p_{1}} \cdot \mathbf{u}_{2 p_{2}}-\frac{i \omega_{c}}{\omega_{3 d}}\left(\frac{k_{2 z}}{\omega_{2 d}}-\frac{k_{1 z}}{\omega_{1 d}}\right) \mathbf{u}_{3 p_{3}}^{*} \cdot\left(\mathbf{u}_{1 p_{1}} \times \mathbf{u}_{2 p_{2}}\right)\right] .
\end{aligned}
$$

The index $s$ denoting particle species has here been dropped for notational simplicity. Furthermore, $F_{0}$ is the unperturbed velocity distribution function, $\omega_{c}=q B_{0} / m$ is the gyrofrequency, $q$ the charge, $m$ the mass, $\omega_{j d}=\omega_{j}-k_{j z} v_{z}-p_{j} \omega_{c}, I_{j}$ $\left(=\exp \left(i \theta_{j}\right)\right)=\left(k_{j_{x}}+i k_{j y}\right) / k_{j \perp}$, and the velocity $\mathbf{u}_{j p_{j}}$ satisfies

$$
\omega_{j d} \mathbf{u}_{j p_{j}}+i \omega_{c} \hat{\mathbf{z}} \times \mathbf{u}_{j p_{j}}=\frac{i q}{m \omega_{j}}\left\{\omega_{j d} J_{p_{j}} \mathbf{E}_{j}+\left[\left(v_{z} E_{j z}+\frac{p_{j} \omega_{c}}{k_{j \perp}^{2}} \mathbf{k}_{j \perp} \cdot \mathbf{E}_{j \perp}\right) J_{p_{j}}+\frac{i v_{\perp} \omega_{c}}{k_{j \perp}^{2}}\left(\hat{\mathbf{z}} \times \mathbf{k}_{j}\right) \cdot \mathbf{E}_{j} \frac{d}{d v_{\perp}} J_{p_{j}}\right] \mathbf{k}_{j}\right\}
$$

where $J_{p_{j}}=J_{p_{j}}\left(k_{j \perp} v_{\perp} / \omega_{c}\right)$ denotes a Bessel function of order $p_{j}$.

The electrostatic limit (where $\mathbf{E}_{j}=-i \mathbf{k}_{j} \Phi_{j}$ ) can be useful if we consider upper-hybrid waves, lower hybrid waves, or electron (or ion) Bernstein waves. In that case, Eq. (4) reduces to ${ }^{7}$ 


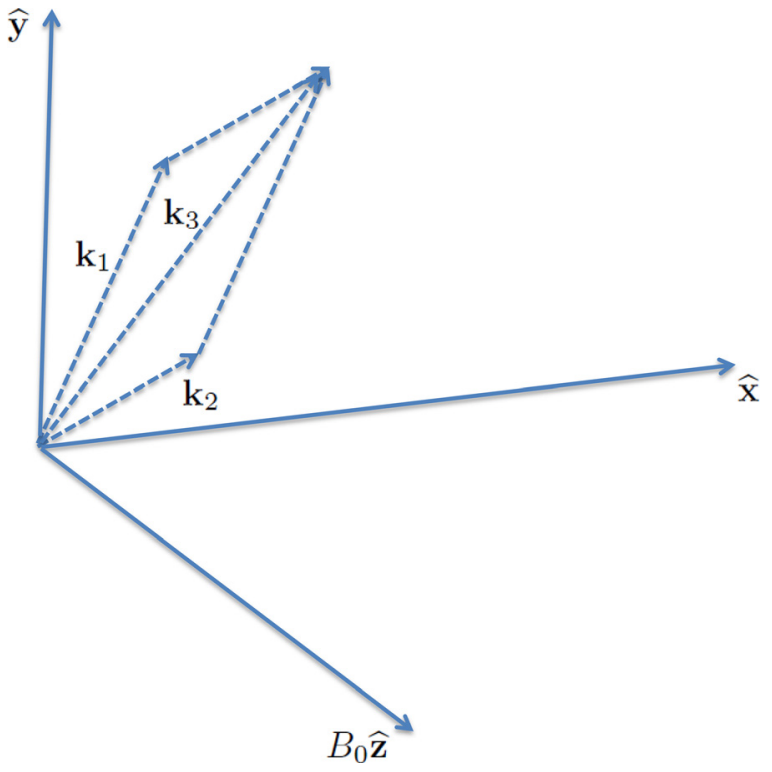

FIG. 1. Schematic figure of the geometry of the problem. Since the magnitude of $\mathbf{k}_{3}$ can be varied independently of $k_{1}$ and $k_{2}$, by varying the angle between $\mathbf{k}_{1}$ and $\mathbf{k}_{2}$, it is obvious that the frequency matching can be fulfilled.

$$
\mathbf{u}_{j p_{j}}=\frac{q \Phi_{j}}{m \omega_{j d}\left(1-\omega_{c}^{2} / \omega_{j d}^{2}\right)}\left(\mathbf{k}_{j}-\frac{i \omega_{c}}{\omega_{j d}} \hat{\mathbf{z}} \times \mathbf{k}_{j}-\frac{\omega_{c}^{2}}{\omega_{j d}^{2}} k_{j z} \hat{\mathbf{z}}\right) J_{p_{j}} .
$$

Below we shall however not limit ourselves to the electrostatic case.

Letting $k_{j z}=0$ for $j=1,2,3$, the last term in the expression (3) for $\mathrm{V}$ disappears. From now on, $\mathbf{k}_{j}$ accordingly denotes general two-dimensional vectors, i.e., $\mathbf{k}_{j}=k_{j x} \hat{\mathbf{x}}+k_{j y} \hat{\mathbf{y}}$, with magnitude $k_{j}=\left(k_{j x}^{2}+k_{j y}^{2}\right)^{1 / 2}$, as is illustrated in Fig. 1. We note that for perpendicular propagation, the velocity $\mathbf{u}_{j p_{j}}$ will either be induced by a wave mode with $\mathbf{E}_{j z}=0$, or by a wave mode with $\mathbf{E}_{j \perp}=0$. This follows from linearized theory if we assume zero net-drift along the magnetic field for all species (i.e., $\int d \mathbf{v} v_{z} F_{0}(\mathbf{v})=0$ ), which we do here.

In the cold limit (see Ref. 13), on which we from now on will focus our interest, we note that only the sums with $p_{j}=0$ contribute. Hence, we have

$$
\mathrm{V}=\sum_{s} m n_{0}\left[\frac{\mathbf{k}_{1} \cdot \mathbf{u}_{1}}{\omega_{1}} \mathbf{u}_{2} \cdot \mathbf{u}_{3}^{*}+\frac{\mathbf{k}_{2} \cdot \mathbf{u}_{2}}{\omega_{2}} \mathbf{u}_{1} \cdot \mathbf{u}_{3}^{*}+\frac{\mathbf{k}_{3} \cdot \mathbf{u}_{3}^{*}}{\omega_{3}} \mathbf{u}_{1} \cdot \mathbf{u}_{2}\right],
$$

where $n_{0}$ is the number density. For the mode with $E_{j z}=0$, we find $\mathbf{u}_{j}$ from

$$
\omega_{j} \mathbf{u}_{j}+i \omega_{c} \hat{\mathbf{z}} \times \mathbf{u}_{j}=\frac{i q \mathbf{E}_{j \perp}}{m}
$$

i.e.,

$$
\mathbf{u}_{j}=\frac{i q}{m\left(\omega_{j}^{2}-\omega_{c}^{2}\right)}\left(\omega_{j} \mathbf{E}_{j \perp}+i \omega_{c} \hat{\mathbf{z}} \times \mathbf{E}_{j \perp}\right),
$$

whereas for the mode with $\mathbf{E}_{j \perp}=0$, we have

$$
\mathbf{u}_{j}=\frac{i q E_{j z}}{m \omega_{j}} \hat{\mathbf{z}}
$$

Finally, for pedagogical reasons, we consider the case of a one-component (electron) plasma. Using linear theory, we express the coupling strengths explicitly in terms of wave amplitudes rather than wave energies. Introducing the electric field amplitudes $E_{j l}=\mathbf{k}_{j} \cdot \mathbf{E}_{j \perp} / k_{j}$, the coupled equations for three extra-ordinary waves are thus

$$
\frac{d E_{3 l}}{d t}=-\frac{1}{\partial D_{\mathrm{eo}}\left(\omega_{3}, k_{3}\right) / \partial \omega_{3}} C E_{1 l} E_{2 l}
$$

and

$$
\frac{d E_{1,2 l}}{d t}=\frac{1}{\partial D_{\mathrm{eo}}\left(\omega_{1,2}, k_{1,2}\right) / \partial \omega_{1.2}} C E_{3 l} E_{2,1 l}^{*},
$$

where

$$
C=\frac{q \omega_{1} \omega_{2} \omega_{3} \omega_{c}^{2}}{m k_{1} k_{2} k_{3}}\left[\frac{k_{1}^{2}}{\omega_{1}} \mathbf{K}_{2} \cdot \mathbf{K}_{3}^{*}+\frac{k_{2}^{2}}{\omega_{2}} \mathbf{K}_{1} \cdot \mathbf{K}_{3}^{*}+\frac{k_{3}^{2}}{\omega_{3}} \mathbf{K}_{1} \cdot \mathbf{K}_{2}\right] .
$$

The dispersion function for the extra-ordinary mode is here

$$
\begin{aligned}
D_{\mathrm{eo}}\left(\omega_{j}, \mathbf{k}_{j}\right)= & \frac{k_{j}^{2} c^{2}\left(\omega_{j}^{2}-\omega_{p}^{2}\right)}{\omega_{j}^{2}} \\
& \times\left[\left(\omega_{j}^{2}-k_{j}^{2} c^{2}\right)\left(\omega_{j}^{2}-\omega_{h}^{2}\right)-\omega_{p}^{2}\left(\omega_{j}^{2}-\omega_{p}^{2}\right)\right],
\end{aligned}
$$

where $c$ is the speed of light, $\omega_{h}=\left(\omega_{p}^{2}+\omega_{c}^{2}\right)^{1 / 2}$ is the upper hybrid frequency, and $\omega_{p}$ is the plasma frequency. Finally, the vectors $\mathbf{K}_{j}$ are

$$
\mathbf{K}_{1,2}=\mathbf{k}_{1,2}+i \frac{\omega_{1,2}}{\omega_{c}}\left(1-\frac{\omega_{p}^{2}}{\omega_{1,2}^{2}}\right) \hat{\mathbf{z}} \times \mathbf{k}_{1,2}
$$

and

$$
\mathbf{K}_{3}=\mathbf{k}_{3}-i \frac{\omega_{3}}{\omega_{c}}\left(1-\frac{\omega_{p}^{2}}{\omega_{3}^{2}}\right) \hat{\mathbf{z}} \times \mathbf{k}_{3} .
$$

The coupling coefficient $C$, defined by Eq. (12), can be written explicitly by carrying out the scalar products, in which case we obtain

$$
\begin{aligned}
C= & \frac{q \omega_{1} \omega_{2} \omega_{3}}{m k_{1} k_{2} k_{3}}\left\{\frac{k_{3}^{2}}{\omega_{3}} \mathbf{k}_{1} \cdot \mathbf{k}_{2}\left[\omega_{c}^{2}-\omega_{1} \omega_{2}\left(1-\frac{\omega_{p}^{2}}{\omega_{1}^{2}}\right)\left(1-\frac{\omega_{p}^{2}}{\omega_{2}^{2}}\right)\right]\right. \\
& \left.+i \omega_{c} k_{3}^{2}\left(\mathbf{k}_{1} \times \mathbf{k}_{2}\right)_{z}\left(1-\frac{\omega_{p}^{2}}{\omega_{1} \omega_{2}}\right)+\text { cycl. perm. }\right\},
\end{aligned}
$$

where cycl. perm. stands for cyclic permutations of $\left(\omega_{1}\right.$, $\left.\omega_{2},-\omega_{3}\right)$ and $\left(\mathbf{k}_{1}, \mathbf{k}_{2},-\mathbf{k}_{3}\right)$. It is easy to see here that the vector nonlinearities (the terms proportional to $\left(\mathbf{k}_{1} \times \mathbf{k}_{2}\right)_{z}$ + cycl. perm.) are in general of the same order of magnitude as the scalar nonlinearity terms (the terms proportional 
to $\mathbf{k}_{1} \cdot \mathbf{k}_{2}+$ cycl. perm.), and thus it plays in general a significant role in any estimate of coupling strength.

We note that in contrast to our previous case, if we instead would have supposed that waves 1 and 2 were ordinary modes with electric field amplitudes $E_{1,2 z} \hat{\mathbf{z}}$ and wave 3 an extra-ordinary mode, the evolution of $E_{1,2 z}$ would have been governed by

$$
\frac{d E_{1,2 z}}{d t}=-\frac{q}{2 m} \frac{k_{3}}{\omega_{2,1}} E_{3 l} E_{2,1 z}^{*} .
$$

Here, we see that in contrast to our previous case, the coupling strength has no explicit dependence on the angles between the wave vectors.

The three wave coupling coefficients play a crucial role for many nonlinear processes. In particular, they determine the threshold values and growth rates for parametric instabilities, see, e.g., Refs. 16 and 17 which constitute key ingredients when studying nonlinear wave absorption. Moreover, weak turbulence theories for plasma waves are typically constructed by summing over all resonant three wave processes and applying the random phase approximation to eliminate the phase dependence. ${ }^{18}$ In the present paper, we have started from the general (but somewhat complicated) kinetic expressions for the coupling strengths. Focusing on the lowtemperature limit with waves propagating perpendicularly to the external magnetic field, we have derived simple formulas for the coupling strengths between three extra-ordinary modes which can be easily applied in concrete situations. A related problem was recently considered in Ref. 12, where the coupling strengths between three extra-ordinary modes were computed. The results were used to construct a onedimensional weak-turbulence theory for extra-ordinary modes. Our expression (16) generalizes the coupling strength to the case where the wave-vectors of the interacting waves are still perpendicular to the magnetic field but in general at different angles. We note that the coupling strength given in Eq. (12) shows an explicit dependence on the angles between the wave vectors. Our result is thus a prerequisite to the construction of a two-dimensional theory of wave turbulence, based on the random phase approximation. For illustrative purposes, we have also commented on the coupling strength when two of the interacting modes are ordinary waves and one wave is an extra-ordinary mode. In this case, the coupling coefficient does not depend on the propagation direction of the waves.

${ }^{1}$ W. L. Kruer, The Physics of Laser Plasma Interactions (Addison-Wesley, New York, 1988).

${ }^{2}$ M. Y. Yu, P. K. Shukla, and R. S. B. Ong, Planet. Space Sci. 35, 295 (1987).

${ }^{3}$ W. A. Scales and H. Xi, Phys. Scr., T84, 184 (2000).

${ }^{4}$ L. Stenflo, Phys. Scr., T107, 262 (2004).

${ }^{5}$ A. Gurevich, Phys. Usp. 50, 1091 (2007)

${ }^{6}$ S. P. Kuo, Phys. Plasmas 22, 082904 (2015).

${ }^{7}$ L. Stenflo, Phys. Scr., T50, 15 (1994).

${ }^{8}$ E. Wallin, J. Zamanian, and G. Brodin, J. Plasma Phys. 80, 643 (2014).

${ }^{9}$ F. Haas and B. Eliasson, Phys. Scr. 90, 088005 (2015).

${ }^{10}$ J. T. Mendonca and A. Serbeto, Phys. Scr., T113, 102 (2004).

${ }^{11}$ M.-J. Lee and Y.-D. Jung, Phys. Plasmas 22, 082105 (2015).

${ }^{12}$ P. H. Yoon, Phys. Plasmas 22, 082310 (2015).

${ }^{13}$ L. Stenflo and G. Brodin, J. Plasma Phys. 72, 143 (2006).

${ }^{14} \mathrm{G}$. Brodin and L. Stenflo, Contrib. Plasma Phys. 54, 623 (2014).

${ }^{15}$ D. G. Swanson, Plasma Waves (Academic Press, New York, 1989), Chap. 4.3 .

${ }^{16} \mathrm{~J}$. Weiland and H. Wilhelmsson, Coherent Non-Linear Interaction of Waves in Plasmas (Pergamon Press, Oxford, 1977).

${ }^{17}$ V. N. Oraevsky, in Basic Plasma Physics Volume 2, edited by A. A. Galeev and R. N. Sudan (North-Holland Physics Publishing, Amsterdam, 1984), Chap. 5.2.

${ }^{18}$ A. A. Galeev and R. Z. Sagdeev, in Basic Plasma Physics Volume 1, edited by A. A. Galeev and R. N. Sudan (North-Holland Physics Publishing, Amsterdam, 1984), Chap. 4. 